\title{
Implicaciones del uso de los Sistemas de Armas Autónomas Letales (Laws) en los conflictos armados modernos
}

Implications of the use of Lethal Autonomous Weapons Systems (Laws) in modern warfare

\author{
Pablo Pugliese ${ }^{2}$ \\ Bautista Griffini ${ }^{34}$
}

\section{Resumen}

En los últimos años, los crecientes desarrollos en el campo de la Inteligencia Artificial se han convertido en uno de los principales motores de innovación tecnológica. Sin embargo, su aplicación en diversos tipos de sistemas de armas comienza a poner de relieve las problemáticas fundamentales de su uso en los escenarios de conflicto tanto actuales como futuros. A partir de un análisis profundo, este trabajo estudia una serie de puntos sobre los cuales la utilización de los Sistemas de Armas Autónomas Letales puede generar controversia. Partiendo desde la tecnología como factor disruptivo en el campo de batalla, pasando por los problemas conceptuales en la definición de la Inteligencia Artificial, recorriendo los dilemas éticos y jurídicos en el uso de estos sistemas, arribando hasta las implicancias de su utilización en el escenario internacional, llegamos a la conclusión de que el uso de estos sistemas traería consigo un proceso de "deshumanización de la guerra".

Palabras clave: Sistemas de Armas Autónomas Letales, Inteligencia Artificial, Deshumanización de la guerra

Recibido: 23 de marzo de 2021 Aceptado: 6 de julio de 2021 Publicado: 8 de julio de 2021

${ }^{1}$ El presente artículo es una versión ampliada y modificada de un trabajo realizado para el Seminario “Tecnología, estrategia y orden internacional" de la Facultad de Ciencias Sociales de la Universidad de Buenos Aires.

${ }^{2}$ Estudiante de la Licenciatura en Ciencia Política. Universidad de Buenos Aires (UBA), Buenos Aires, Argentina. Miembro del Centro de Estudios de Política Internacional (UBA), y del Grupo de investigación en Política Exterior Argentina. Correo electrónico: pablo-pugliese@hotmail.com ID https://orcid.org/00000001-9111-6540

3 Estudiante de la Licenciatura en Ciencia Política. Universidad de Buenos Aires (UBA), Buenos Aires, Argentina. Miembro del Centro de Estudios de Política Internacional (UBA), y del Grupo de Investigación en Política Exterior Argentina. Correo electrónico: bauti_griffini@hotmail.com ID https://orcid.org/00000003-3124-043X

${ }^{4}$ Los autores agradecen las acertadas observaciones y sugerencias realizadas por uno/a de los evaluadores/as anónimos. 


\section{Abstract}

In recent years, the growing developments in Artificial Intelligence have become one of the main sources of technological innovation. However, its application in various types of weapons systems begins to highlight the fundamental problems of its use in current and future conflict scenarios. Starting from a deep analysis, this work studies a series of points on which the use of Lethal Autonomous Weapons Systems can generate controversy. From technology as a disruptive factor on the battlefield, through the conceptual problems in defining Artificial Intelligence, the ethical and legal dilemmas in the use of these systems, to the implications of their use in the international system, we conclude that the use of these systems would bring about a process of "dehumanization of war".

Keywords: Lethal Autonomous Weapons Systems, Artificial Intelligence, Dehumanization of war

\section{Introducción}

Numerosas historias -tanto del ámbito cinematográfico como del literario- han abordado recurrentemente la idea de un futuro donde el desarrollo de las máquinas adquiriera un nivel suficiente para reemplazar al hombre en todo tipo de actividades, incluso al punto de llegar a dominar y someter a la propia civilización humana. Desde la aparición de las obras de autores como Isaac Asimov, o las películas como Blade Runner de Ridley Scott, Matrix de las hermanas Wachowski y Terminator de James Cameron, el concepto de "rebelión de las máquinas" ha sido motivo de preocupación constante y ha permitido poner de relieve las problemáticas derivadas del desarrollo tecnológico y las posibles consecuencias no deseadas que el mismo pueda traer consigo. ${ }^{5}$

En la actualidad, los cada vez más frecuentes usos de la Inteligencia Artificial comienzan a ser aplicados en todo tipo de ámbitos, desde los artefactos más comunes hasta en armamentos de los más variados. Países como China, Israel, Corea del Sur, Rusia, Reino Unido, Turquía y Estados Unidos han proyectado gran parte de su presupuesto en investigaciones en esta área, buscando desarrollar todo tipo de

\footnotetext{
${ }^{5}$ La idea de la alusión no es ni mucho menos banalizar la discusión realizando una comparación entre Terminator y las Laws, lejos queremos estar de ello y sería erróneo plantearlo de esa manera, sino más bien dejar de manifiesto cómo la implementación de la tecnología puede traer consecuencias no deseadas.
} 
aplicaciones militares integradas con estas tecnologías, que se convierten cada vez más en un punto estratégico de sus políticas de defensa. ${ }^{6}$

En este sentido, la irrupción de la Inteligencia Artificial aplicada promete generar un punto de inflexión en los nuevos desarrollos armamentísticos, dando origen a una suerte de proceso de deshumanización en las operaciones de guerra, al dotar a dichos sistemas de autonomía en la toma de decisiones respecto del escenario de conflicto en los cuales se desenvuelven, motivo por el que la utilización de los mismos se ha convertido actualmente en objeto de debate por parte de las comunidades científicas y las principales organizaciones internacionales de derechos humanos. A partir de dicha problemática, comienza a delinearse una de las cuestiones más discutidas de la actualidad en el ámbito de la Defensa y la Seguridad Internacional: las implicaciones derivadas de la utilización de los denominados "Sistemas de Armas Autónomas Letales" (Laws por sus iniciales en inglés) en los conflictos armados modernos.

El propósito de esta investigación será exponer las dificultades relativas al uso de la Inteligencia Artificial aplicada, como así también elaborar una investigación exhaustiva referente a los problemas y dilemas (éticos, jurídicos, políticos y técnicos) derivados de la utilización de las Laws en los conflictos armados modernos y futuros. La primera parte apuntará a exponer la importancia del factor tecnológico como elemento disruptivo y de superioridad en el campo de batalla. La segunda parte estará dirigida a abordar los problemas referentes a la Inteligencia Artificial, señalando los límites y ambigüedades de su definición, como así también las ventajas derivadas de su uso. La tercera parte estará abocada a analizar los riesgos y dilemas éticos que representa la aplicación de la Inteligencia Artificial en la categoría de los denominados Sistemas de Armas Autónomas Letales (Laws). La cuarta parte, a su vez, estará dedicada a poner de relieve la reglamentación de su utilización derivada del Derecho Internacional y los tratados vigentes, como así también los conflictos y "lagunas jurídicas" que surgen de los mismos, junto a las distintas campañas e iniciativas existentes para su regulación y control. La quinta parte se orientará a brindar una perspectiva sobre el impacto de este tipo de tecnologías en el escenario internacional.

\footnotetext{
${ }^{6}$ El STM Kargu-2 es un ejemplo muy claro. Fabricado en Turquía por la compañía STM, el mismo se lleva comercializando desde hace cerca de dos años y puede ser utilizado con eficacia contra objetivos estáticos o en movimiento a través de sus capacidades de procesamiento de imágenes en tiempo real y los algoritmos de aprendizaje automático integrados en la plataforma.

${ }^{7}$ A pesar de que el día de hoy no existe un consenso acerca de la definición certera con respecto a los sistemas de armas autónomas letales, en términos generales y siguiendo el planteo de la reunión de expertos pronunciada por la Cruz Roja Internacional del 26 al 28 de marzo de 2014 en Ginebra (Suiza), podemos identificar a los mismos como aquellos sistemas de armas que cuentan con la autonomía suficiente para seleccionar y atacar objetivos de manera independiente.
} 
Finalmente, se elaborarán las conclusiones, poniendo especial énfasis en las posibles consecuencias y desafíos a futuro por parte de los diversos actores del escenario internacional.

\section{El factor tecnológico como elemento disruptivo en el campo de batalla}

Desde la antigüedad, el uso de la tecnología ha sido un factor fundamental en el progreso de la humanidad, al permitir aumentar de manera significativa la producción de bienes a un costo cada vez menor, promoviendo consecuentemente un creciente acceso a los mismos. Sin embargo, el desarrollo de tecnologías innovadoras también ha traído consigo una gran variedad de problemas: como afirma Fritsch (2017), por un lado, las mismas se han convertido en un poderoso motor de cambio en el desarrollo económico, político, militar y cultural de la sociedad global, mejorando las condiciones de vida de millones de personas, pero, por otro, han multiplicado la capacidad destructiva y el alcance global de los conflictos armados.

Queda claro que, a lo largo de la historia, la superioridad tecnológica en el campo militar ha sido un elemento clave para alcanzar la victoria en el campo de batalla, como también para garantizar la conquista y la dominación de un pueblo sobre otro. Quizás los ejemplos más representativos de ello fueron, por una parte, la conquista de América por parte de España, donde armas tales como arcabuces y trabucos fueron esenciales para garantizar la desmedida ventaja de los europeos por sobre los pueblos nativos, que solo disponían de lanzas, arcos y flechas para defenderse; y, por otra, los ataques nucleares sucedidos en Japón en 1945. Esto explica, en gran medida, el importante rol estratégico que los actores estatales le han asignado a estos desarrollos, razón por la cual han sido impulsados desde las más altas esferas de la política.

Desde las innovaciones atómicas ejecutadas al final de la Segunda Guerra Mundial, el potencial de destrucción ha llegado a su plenitud y se ha convertido en una amenaza global. El desarrollo tecnológico expone así su peligrosidad siempre latente, abonando la tesis de Headrick (2011) de que, aunque ninguna tecnología obligue a las personas a utilizarla, cualquier nueva forma de poder sobre la naturaleza despierta la tentación de hacerlo, a pesar de los riesgos derivados.

En la actualidad, la Inteligencia Artificial comienza a delinearse como un desarrollo fundamental y de carácter estratégico a nivel mundial. La integración de este tipo de tecnología en diversos armamentos ya no es mera ciencia ficción, sino que se encuentra en pleno auge. En los últimos años, han venido creciendo el uso de estos sistemas, principalmente en su variante aérea, conocida bajo la categoría de Vehículos Aéreos No Tripulados de Combate (UCAV por sus siglas en inglés) en 
conflictos a lo largo del mundo. Si bien al día de hoy no existe un UCAV completamente autoconsciente, los desarrollos en este campo son cada vez más evidentes, y pueden observarse en el sentido que señala Sloan (2015), con la reconversión de los actualmente utilizados "sistemas semiautónomos"(autodirigidos), que están programados para realizar acciones específicas, las cuales llevan a cabo independientemente de influencias o control externo, hacia los denominados "sistemas autónomos" (autodeterminados), que en lugar de tener una respuesta preprogramada buscan la solución óptima en situaciones imprevistas, eligiendo su objetivo, adaptándose y aprendiendo de la información percibida a su alrededor, de manera que son capaces de responder a su entorno de diferentes formas, dependiendo de las circunstancias (p. 114).

$\mathrm{Al}$ respecto, si bien numerosos especialistas se oponen a ello, las ventajas de la utilización de este tipo de sistemas no son pocas, ya que permiten obtener una relativa superioridad militar en el teatro de operaciones, un menor costo tanto económico como político (ya que evitan algunas de las consecuencias más dramáticas de la guerra), un menor tiempo de reacción con respecto al de los seres humanos, y la obediencia incondicional por parte de la máquina ante cualquier situación (siempre que esto no falle). Esto equivale a decir que con la utilización de estas tecnologías disminuyen las pérdidas humanas, y que además estos sistemas pueden ser incluso más eficientes que un piloto humano en realizar tareas, minimizando el daño colateral.

Esta suerte de eficientización del conflicto derivado del aumento de la tecnología es vista por muchos como el futuro escenario de una guerra más cruel y deshumanizada, mientras que otros expertos señalan lo contrario. Siguiendo el planteo, tanto la visión optimista, que ve en este tipo de tecnologías el futuro del combate, como la pesimista, que ilustra fielmente los horrores del progreso tecnológico a partir de los cuales, como señala Beck (2009), los humanos no somos capaces de controlar nuestras propias creaciones ni de prever sus consecuencias -por lo que nos convertimos en la fuente de nuestra propia destrucción-, nos permiten afirmar que cada nueva innovación supone riesgos incalculables, y que la tecnología debería dejar de tener un "cheque en blanco".

\section{Los problemas conceptuales en la definición de la Inteligencia Artificial}

En los actuales debates que se erigen con respecto a la Inteligencia Artificial, existe un problema fundamental en cuanto a la elaboración de una definición adecuada que permita una delimitación efectiva en cuanto a qué es y qué no es una Inteligencia Artificial. No existe un consenso aún para responder a esta pregunta y de 
ahí las dificultades que ello representa. Una Inteligencia Artificial puede definirse tanto como una forma de vida artificialmente creada que sobrepase los límites de la inteligencia humana, como también para referirse a cualquier tecnología que procese un conjunto de datos a partir de un determinado aprendizaje.

En líneas generales, el término se encuentra en una permanente resignificación, dado que a lo largo del tiempo ha ido evolucionando, es decir, que tecnologías que hace 50 años atrás podrían haber sido consideradas como Inteligencia Artificial, hoy ya no lo son, ya que se han convertido en parte de nuestra vida cotidiana y su uso se ha hecho cada vez más común.

Una primera aproximación al concepto actual de Inteligencia Artificial nos lleva a la definición de dos elementos principales en los que ella se enmarca. Por un lado, el concepto de "autonomía", entendida como la habilidad de llevar adelante diversas funciones en entornos complejos sin ningún tipo de intervención humana, y, por otro, la idea de "adaptabilidad", como la habilidad de mejorar la performance a partir del aprendizaje derivado de la experiencia.

A partir de estas discusiones, podremos finalmente distinguir cuatro maneras de abordar las definiciones de Inteligencia Artificial de manera más efectiva. Por un lado, tendremos la distinción entre "Inteligencia Artificial General" (AGI), entendida como aquella a partir de la cual la máquina puede realizar cualquier tipo de tarea intelectual; y por otro, la "Inteligencia Artificial Estrecha" (ANI), como aquella que sólo puede ocuparse de una tarea determinada y especializada. Por otra parte, para saldar la discusión filosófica en cuanto al uso de estas tecnologías, tendremos la diferenciación entre "Inteligencia Artificial Fuerte" (SAI), como aquella que dispone de genuina inteligencia y autoconciencia, y, por otra parte, la "Inteligencia Artificial Débil” (WAI), que es la actualmente existente y consiste en sistemas que exhiben un comportamiento inteligente limitado para realizar tareas específicas.

Esto se puede relacionar directamente con el grado de autonomía o control que pueden tener las Laws. Según Martinez y Rodríguez (2019) existen cuatro tipos de control: "human full control", donde el sistema no toma ninguna decisión por sí mismo sino que es teledirigido; "human in the loop", en donde el sistema implementa la tarea ordenada con autonomía, pero requiere la intervención humana para validar e implementar acciones; "human on the loop", en donde el sistema implementa la tarea ordenada en autonomía bajo la supervisión de operadores humanos que pueden, si es necesario, corregir o abortar una acción específica y "human off the loop", aquellas en el que el sistema de armas desarrolla una tarea programada $u$ ordenada sin supervisión o intervención de operadores del Sistema de Comando y Control, conforme a sus capacidades y condiciones de diseño. (p. 6). Para que nos quede claro hay que mencionar que en la actualidad no existen armas totalmente autónomas, sino 
que está garantizado algún tipo de control. Sin embargo, esta situación está cercana a cambiar.

\section{Los dilemas éticos en el uso de los Sistemas de Armas Autónomas Letales}

El actual crecimiento en el desarrollo de sistemas de armas autónomas por parte de diversos actores estatales abre un abanico de interrogantes en torno a los dilemas éticos que acarrea su implementación en el teatro de operaciones. En este sentido, el dominio de estas innovadoras capacidades armamentísticas cambia casi por completo y modifica constantemente la doctrina militar a un ritmo sin precedentes, llevando consecuentemente a una dinámica cada vez más deshumanizada de los conflictos en términos de un creciente reemplazo del combatiente humano. ${ }^{8}$

Cabe mencionar en este sentido que, a lo largo de la historia, filósofos como Carl Schmitt, Theodor Adorno y Hannah Arendt han vislumbrado la relación entre el desarrollo tecnológico y la ferocidad de la guerra. Keegan (2013), a su vez, mencionaba que con la llegada de la industrialización habían cambiado los ritmos de los conflictos bélicos, de manera que las batallas pasaron a adquirir una dinámica mecanicista, predominando una suerte de lógica matemática en beneficio de administrar los recursos de la manera más eficaz posible, a los fines de alcanzar la victoria (p.225).

Siguiendo esta línea, la idea de Adorno (2001) de que, a mayor innovación tecnológica menor faceta humana, se ve complementada por el abordaje de Schmitt (2005), quien es pionero en advertir una correlación entre el mayor perfeccionamiento de las armas y el carácter discriminatorio y moralizante de la guerra.

El planteo de Arendt (2006), que concibe que la edad contemporánea está marcada por guerras y revoluciones favorecidas por el desarrollo técnico de los medios de la violencia, que acrecientan a la vez el potencial destructivo, estando por primera vez dentro de las capacidades humanas la destrucción de la civilización misma, es totalmente esclarecedor y complementario respecto de las consideraciones del mencionado Schmitt (2005), que veía el dominio del aire -con el advenimiento de la aviación de guerra- como un elemento que contribuía a la deshumanización de la batalla, por cuanto consideraba que el fin del ataque aéreo era meramente destructivo, como también una demostración de fuerza. Para el mismo autor, el hecho de que el atacante aéreo esté demasiado lejos de la tierra como para entablar un contacto con

\footnotetext{
${ }^{8}$ Muchas de las argumentaciones realizadas en este apartado, son consideraciones tomadas a partir del empleo de drones en el teatro de operaciones. Las Laws profundizarían -en gran medida- la mayoría de los dilemas éticos de la utilización de los sistemas anteriormente mencionados.
} 
el adversario, supone una situación asimétrica y de superioridad, de manera que contra el enemigo cualquier medio pasa a ser válido y por ello la guerra ya no puede ser acotada, sino total.

En la actualidad, la introducción de armas aéreas autónomas consolida un avance adicional en este sentido, modificando de manera completa el concepto del espacio bélico, tanto a partir de la criminalización del enemigo, que es tomado como mero blanco, como a partir de la desvinculación física y humana que supone la máquina, situaciones que cambian a la vez las condiciones de percepción de la batalla. En este sentido, el enfoque de "guerra remota" elaborado por los Estados para contrarrestar las amenazas a distancia nos resulta esclarecedor. En lugar de desplegar un gran número de sus propias tropas, los países utilizan una variedad de tácticas para apoyar a los socios locales que realizan la mayor parte de los combates de primera línea. Es importante destacar que la guerra remota no se lleva a cabo únicamente a través de sistemas remotos, concepto que a veces se denomina "remote control war" (Gusterson, 2016), sino que las tecnologías remotas desempeñan un papel, pero ese tipo de guerra abarca un conjunto más amplio de acciones. En este sentido, la implementación de Laws se puede entender dentro de dicho enfoque.

Siguiendo a Sandoval (2018), ante todo, la implementación de las Laws conlleva la desaparición de la noción clásica de "campo de batalla" como el lugar acotado y delimitado donde tiene lugar el conflicto y rige el ius belli. El campo ahora es la tierra entera, con la consecuente caducidad de la noción de "zona de conflicto armado" interpretada en sentido geográfico (p.174-175). Por lo tanto, comienza a entrar en juego una dislocación espacial (múltiples espacios), tanto física como virtualmente.

Nos resultan particularmente interesantes los conceptos que utiliza Ryan (2014) de "disociación" en el sentido de la falta de compromiso que surge en la medida en que la distancia entre beligerantes aumenta desde tres puntos a considerar: (1) disociación de los agentes (burocracia militar) respecto de sus actos violentos; (2) disociación de los objetivos enemigos respecto de la fuente de violencia dirigida contra ellos; (3) disociación del público (población) respecto de la violencia cometida en su nombre (p.223).Y, junto a ello, el concepto de "re espacialización” de Gusterson (2014), referido a un desdoblamiento o multiplicación del espacio, también es muy útil (p.197). Se abren en este sentido dos ámbitos distintos: por un lado, el espacio de acción donde opera el sistema autónomo (campo de batalla) y, por otro, el espacio de dirección (pacificado), desde donde se le brindan las misiones y órdenes. Estos ámbitos representan dos realidades distintas desde el punto de vista de la vivencia de la guerra.

Siguiendo esta consigna, podemos considerar que la utilización de Laws en el teatro de operaciones produce en el enemigo una privación de la posibilidad de 
respuesta con respecto al atacante. En esta línea, consideramos que la distancia que separa a la máquina autónoma de su objetivo facilita en gran medida la administración de la violencia y se traduce, asimismo, en una asimetría o falta de proporcionalidad entre ataque y posibilidad de respuesta.

Entonces, desde el punto de vista ético, podemos inferir que el enfrentamiento no es recíproco, sino unilateral; la guerra se convierte en un mero ejercicio de caza.

\section{Los problemas y desafíos jurídicos en torno a la utilización de los Sistemas de Armas Autónomas Letales}

Uno de los aspectos más interesantes que se pierden de vista frente a la utilización de estos instrumentos corresponde al dilema jurídico de asignación de responsabilidades derivadas de las posibles fallas de las Laws en el combate. Frente a ello, surgen diversas preguntas en torno a un posible mal funcionamiento o error de estas: ¿quién sería el responsable de ello?, ¿es el sistema en sí?, ¿es su programador?, ¿quién elabora los algoritmos que rigen su funcionamiento?, ¿es el comandante militar que da la orden de utilizarlo en las misiones?, ¿es el político que se encuentra detrás de la implementación del diseño? El origen de la responsabilidad internacional en sentido clásico lo constituye el hecho internacionalmente ilícito. Podemos definir el hecho internacionalmente ilícito como un hecho atribuible a un sujeto jurídicointernacional que, constituyendo una violación o infracción del Derecho internacional, lesiona derechos de otro sujeto u otros sujetos de dicho ordenamiento, o incluso derechos o intereses de los que sería titular la propia comunidad internacional, dando lugar, entre otras consecuencias, a la responsabilidad del sujeto autor del hecho. La consecuencia jurídica más característica y normal del hecho internacionalmente ilícito es la responsabilidad internacional del sujeto a quien el hecho es atribuible y suele concretarse en el deber de reparar. ${ }^{9}$ Lo cierto es que hasta el día de hoy no existen respuestas consensuadas frente a estos interrogantes en el Derecho Internacional sobre quién sería el responsable frente a un error con las LAWS.

La idea de que en una guerra las acciones no deben separarse de sus consecuencias, ilustra la problemática que se erige en torno a la utilización de este tipo de armamento en el campo de batalla. Todo delito involucra un acto y un estado mental (intención), que quedan completamente diluidos en términos de imputabilidad en el caso de que el ejecutor sea una máquina autónoma.

\footnotetext{
${ }^{9}$ Artículo 1 del proyecto de artículos de la Comisión de Derecho Internacional de la ONU sobre la responsabilidad del Estado por hechos internacionalmente ilícitos.
} 
Al respecto, Sandoval (2018) señala que la utilización de estos sistemas supone una ausencia de derechos por parte del objetivo, en tanto el enemigo carece de derecho a ser enemigo y por ende a ser tratado como prisionero de guerra; y, por otro lado, agrega que existe una falta de responsabilidad por parte del lado atacante, ya que no hay sujeto a quien imputar dicha responsabilidad (p. 177).

En este sentido, existen numerosos interrogantes también en torno a las vulneraciones a la normativa vigente que este tipo de dispositivos puedan llegar a ocasionar en las operaciones en que intervengan, fundamentalmente en lo que hace a aquellas premisas consagradas y codificadas en los diversos pactos y tratados del Derecho Internacional Humanitario y los Derechos Humanos que rigen el ius in bello. Más específicamente, las referidas al "principio de distinción" ${ }^{10}$, que apunta a la diferenciación entre objetivos lícitos y aquellos que no lo son, en virtud de proteger a la población civil de ataques indiscriminados; y el "principio de proporcionalidad" 11 , que implica una evaluación previa del daño que pueda causarse a una población no combatiente respecto de la ventaja militar que el ataque pretende conseguir; este principio conlleva una instancia de decisiones cualitativas que actualmente solo el humano puede tomar, en tanto dependen en gran medida del contexto y de las circunstancias de combate, que escapan a la programación de un sistema autónomo. ${ }^{12}$ También es menester mencionar el "principio de precaución" contra los efectos de los ataques, en donde "las partes en conflicto deberán tomar todas las precauciones factibles para proteger de los efectos de los ataques a la población civil y los bienes de carácter civil que estén bajo su control”.

Como resultado de estas discusiones, en los últimos años han surgido diversas campañas ${ }^{13}$, con el fin de poner de relieve los problemas derivados de la utilización de este tipo de sistemas, como por ejemplo Campaign to Stop Killer Robots, e iniciativas de diversas instituciones, como el Comité internacional de la Cruz Roja y Media Luna Roja, que buscan un instrumento jurídicamente vinculante que prohíba expresamente cierto tipo de armas. En líneas generales, existe un consenso sobre la necesidad de evitar el uso dual de la Inteligencia Artificial aplicada, a partir del concepto de "control humano significativo" como elemento central en la autonomía de todo sistema de armas. Si bien existe un debate en torno a la interpretación de esta

\footnotetext{
${ }^{10}$ Consagrado en el Artículo 48 del Protocolo Adicional I de las Convenciones de Ginebra.

${ }^{11}$ Consagrado en el Artículo 51 (5) (b) del Protocolo Adicional I de las Convenciones de Ginebra.

${ }^{12}$ Cabe señalar que, junto al principio de necesidad militar, tanto el principio de distinción como el principio de proporcionalidad conforman el conjunto de los tres principios más importantes del Derecho Internacional Humanitario que legislan el uso de la fuerza en un conflicto armado.

${ }^{13}$ Creemos fervientemente en la necesidad de contar con estas iniciativas desde la sociedad civil; sin embargo, sostenemos que una vez que los Estados comienzan -de manera efectiva- a pensar seriamente en la utilización de estas tecnologías, resulta muy difícil frenar su implementación.
} 
idea (United Nations Institute for Disarmament Research [UNIDIR], 2014) ${ }^{14}$, el mismo toma el concepto acuñado por Article 36, una aproximación que enfatiza la calidad del control de los sistemas de armas autónomos letales y supone la delegación de la responsabilidad en agentes humanos de toda decisión concerniente a la ejecución de ataques letales sobre individuos.

$\mathrm{El}$ argumento central de todos estos planteos se fundamenta principalmente en que la utilización de las Laws atenta contra el cumplimiento del principio de dignidad humana a partir de la delegación de la decisión de vida o muerte en la máquina y, por otro lado, también en que reducen la rendición de cuentas (accountability) desde el punto de vista político-jurídico. Desde esta perspectiva, Docherty \& Griechen (2019) destacan la importancia de la denominada "Cláusula Martens" ${ }^{15}$, por cuanto la misma vincula el derecho a la ética, declarando que, ante la ausencia de un tratado específico con respecto a un tema, las personas continúan estando protegidas por la costumbre, por los principios humanitarios y por el dictado de la conciencia pública en términos de lineamientos morales compartidos (p. 7-9).

\section{El uso de los Sistemas de Armas Autónomas Letales y su impacto en el Escenario Internacional}

Durante el año 2014, el entonces Secretario de Defensa de los Estados Unidos, Chuck Hagel, señaló que los rápidos avances de la inteligencia artificial definirían la próxima generación de la guerra, en el marco de la anunciada Tercera Estrategia de Compensación. ${ }^{16}$ Siguiendo esta línea, en los últimos tiempos comienzan a hacerse cada vez más frecuentes los desarrollos y la utilización de estos sistemas en conflictos de baja escala a lo largo del mundo. La incorporación de Laws a las fuerzas armadas de numerosas naciones supone la utilización de una tecnología de punta cuyas ventajas son innumerables a la hora de ejecutar misiones de todo tipo.

La paulatina incorporación e implementación de estos instrumentos nos permiten contrastar de forma nítida las diferencias entre un "soldado humano", caracterizado por una amplia perspectiva situacional y una capacidad empática derivada de su conocimiento de la muerte, y un "soldado robot", caracterizado por una mayor rapidez y precisión en términos de eficiencia. En este sentido, resulta importante señalar que el comportamiento de las Laws está signado en gran medida

\footnotetext{
${ }^{14}$ Instituto de las Naciones Unidas para la Investigación del Desarme, UNIDIR por sus iniciales en inglés.

${ }^{15}$ Introducida en los preámbulos de la Segunda convención de La Haya sobre Leyes y Costumbres de la Guerra Terrestre de 1899.

${ }^{16} \mathrm{Al}$ respecto, Colom (2015) señala que la Tercera Estrategia de Compensación consiste en una iniciativa para incrementar la brecha de capacidades entre Estados Unidos y sus potenciales adversarios, garantizando la capacidad de proyectar poder en cualquier punto del planeta, con independencia de las medidas anti-acceso y denegación de áreas por parte del enemigo.
} 
por su evolución y su aprendizaje (machine learning), y estos son precisamente los elementos que las vuelven imprevisibles. Teniendo en cuenta esto, podemos señalar la idea de que los seres humanos son mejores juzgando situaciones ambiguas y complejas, mientras que las máquinas sólo superan al humano en funciones especializadas, en tanto "piensan" en sentido limitado.

Por otro lado, uno de los principales problemas en lo que hace al tratamiento y la regulación de estos sistemas en el plano institucional internacional, surge a partir de las diferentes concepciones de autonomía, que dan lugar a la categorización y delimitación de aquello que se considera un "sistema de armas autónomo letal" por parte de los distintos actores estatales. A continuación, siguiendo el informe de PAX (2019) "State of $A I$ ", ofrecemos una comparación entre los países que cuentan con desarrollos considerables en inteligencia artificial, priorizando aquellos que prestan particular atención a sus usos con fines militares. Al respecto, decidimos incluir al Vaticano, no desde ya por su desarrollo o uso de Laws, sino debido a que es un Estado referencial en lo que respecta a la ética de la guerra y el uso de la fuerza.

China, en líneas generales, mantiene una posición ambivalente: por un lado, adhiere a una prohibición completa de las Laws, pero sólo respecto de su uso en el campo de batalla, y no en cuanto a su desarrollo o producción. Simultáneamente, desarrolla este tipo de tecnología, buscando así utilizar la ley internacional en contra de sus competidores.

En el documento del Group of Governmental Experts of the High Contracting Parties to the Convention on Prohibition or Restrictions on the Use of Certain Conventional Weapons which may be deemed to be Excessively Injurious or to have Indiscriminate Effects, se caracteriza a las Laws a partir de su "letalidad", referida a su capacidad de daño; de su "autonomía", entendida como la ausencia de intervención humana y de control durante el proceso completo de ejecución de las tareas; de sus "efectos indiscriminados", referidos a llevar a cabo tareas que demanden la ejecución o mutilación independientemente de las condiciones, escenarios y conflictos; y de su "evolución", entendiendo como tal la interacción con el entorno, por cuanto el dispositivo puede aprender de forma autónoma, aumentando sus funciones y capacidades de una manera que exceda las expectativas humanas.

Estados Unidos -por su parte-, en el documento Departament of Defense Directive [DoDD] (2012), define las Laws (basándose en el rol del operador humano con respecto a la selección de objetivos y en el compromiso del mismo en la toma de decisiones) como aquellos sistemas de armas que, una vez activados, pueden seleccionar y atacar objetivos sin intervención adicional de un operador humano (concepto de human out of the loop ofull autonomy). ${ }^{17}$

\footnotetext{
${ }^{17}$ Recuérdese que la discusión sobre los grados de autonomía fue mencionada en el apartado dos.
} 
La política de Gran Bretaña (en cuanto a las Laws) se fundamenta en dos publicaciones: el Joint Doctrine Note 2/11 (2011) y la Joint Doctrine Publication 0-30.2. (2017). En ellos, distingue entre "sistemas automatizados" (aquellos sistemas autoconscientes con capacidad de alcanzar un nivel de entendimiento situacional igual o mayor al del humano) y "sistemas autónomos" (aquellos que siguen lógicamente un conjunto predefinido de reglas o instrucciones y que no son capaces de alcanzar niveles de entendimiento situacional). Desde esta perspectiva, las Laws como tales no existirían, sino que hoy en día sólo existirían sistemas armados automatizados. Hay posicionamientos de los países, más recientes, de todos los países

Francia -basándonos en su posición ante las Naciones Unidas- ${ }^{18}$ entiende a las Laws de forma bastante avanzada y "futurista": las mismas sería total, es decir que no habría ninguna forma de supervisión humana desde el momento de la activación, y ninguna subordinación a una cadena de mando.

Por su parte, la posición oficial de Rusia respecto a las Laws se fundamenta en la consideración de la autonomía a base de la idea de "singularidad" o "superinteligencia”. Desde este enfoque, la plena autoconciencia de la máquina aún está lejos de existir. El Diccionario Enciclopédico Militar del Ministerio de Defensa de Rusia (2020) denomina "robot de combate" a todo dispositivo técnico multifuncional con un comportamiento antropomórfico, capaz de desempeñar total o parcialmente las funciones propias que desarrollaría un humano en misiones de combate. En esta línea, Rusia distingue tres generaciones de robots de combate: los de Primera Generación serían aquellos robots que disponen de software y control remoto, y que sólo pueden operar en entornos organizados; los de Segunda Generación serían robots capaces de adaptarse a cambios en su entorno, los cuales poseen instrumentos de censado y capacidad de funciones en ambientes aleatorios; por último, los de Tercera Generación serían aquellos robots inteligentes equipados con sistemas de control basados en Inteligencia Artificial.

En lo que hace a los casos de Israel y Corea del Sur no se encuentran definiciones claras en lo que respecta a las Laws; sin embargo, podemos reconocer dichas posiciones gracias a sus posturas manifestadas en organismos internacionales. En la reunión de la ONU llevada a cabo en $2018^{19}$ Israel declaró que el "desarrollo de estándares rígidos, o la imposición de prohibiciones de algo que es tan especulativo en esta etapa temprana, sería imprudente y podría producir un resultado mal

\footnotetext{
${ }^{18}$ Convention on Prohibitions or Restrictions on the Use of Certain Conventional Weapons Which May Be Deemed to Be Excessively Injurious or to Have Indiscriminate Effects.

19 Ambas posiciones fueron manifestadas en el "Group of Governmental Experts on Emerging Technologies in the Area of Lethal Autonomous Weapons System".
} 
informado y equivocado". ${ }^{20}$ En el mismo sentido, la comitiva subrayó que "nosotros también deberíamos ser conscientes de la situación militar y humanitaria y las ventajas que se pueden asociar a las Laws, tanto desde el punto de vista operativo, legal y ético; estas ventajas pueden incluir una mejor precisión de la focalización, que minimizaría el daño colateral y reduciría riesgos para los combatientes y no combatientes". Por su parte, Corea del Sur, a diferencia de Israel, no hizo declaraciones en el grupo de expertos que se reunió en 2018. No obstante, podemos rastrear declaraciones anteriores; por ejemplo, una que realizó en 2015, donde sostiene que "las discusiones sobre leyes no deben llevarse a cabo de una manera que pueda obstaculizar la investigación y el desarrollo de tecnología robótica para uso civil ", pero que "desconfía de la autonomía total de los sistemas de armas que eliminan el control humano significativo del ciclo de operaciones, debido al riesgo de mal funcionamiento y posibles brechas de responsabilidad y preocupaciones éticas".

Finalmente, el Vaticano en la Convención de Ginebra (2019) define las Laws como todo sistema de armas capaz de identificar, seleccionar y realizar una acción sobre un blanco determinado sin supervisión humana. En esta línea, clasifica distintos niveles de autonomía, según: 1) el grado y la duración de la supervisión humana, 2) la previsibilidad del comportamiento del robot y 3 ) las características del medio en el que opera, que incluye sus límites geográficos y sus objetivos acerca de personas, objetos y eventos probables.

Sin embargo, y guiándonos por el documento "Group of Governmental Experts on Emerging Technologies in the Area of Lethal Autonomous Weapons System" (2021) podemos vislumbrar una gran preocupación por parte de los países con relación al respeto del Derecho Internacional Humanitario y mantener un control humano significativo en las Laws.

Más allá de las disimilitudes en la definición de los sistemas de armas autónomas, otro de los dilemas relativos al uso de la Inteligencia Artificial aplicada en armas surge a partir de la cantidad de datos que dichos sistemas absorben en su proceso de aprendizaje, y que se circunscriben a un contexto determinado, lo que equivale a decir que la base de datos es limitada y representa solo una parte de la totalidad de posibilidades reales. En ese sentido, es preciso recordar que los datos que recogen las Laws durante su aprendizaje son decisivos en su posterior comportamiento. Sumado a ello, el procesamiento de los datos seleccionados también

\footnotetext{
${ }^{20}$ Inmediatamente uno observa la posición de Israel, nota una tendencia bastante favorable al uso de estos sistemas. Esto puede deberse al carácter específico de los conflictos en los que Israel se encuentra actualmente. Por ejemplo, uno de los métodos más efectivos para contrarrestar la cercanía respecto a los ataques de Hamas podría ser utilizando dichos sistemas, tanto defensivamente como ofensivamente. Un ejemplo parcial podría ser el sistema móvil "Cúpula de Hierro", un sistema semiautónomo que permite interceptar misiles de corto alcance (de 4 a 70 kilómetros).
} 
está sesgado por la programación de la máquina, y por ende estarán sesgadas también sus conclusiones y decisiones.

En este sentido, el funcionamiento de las máquinas autónomas se ajusta a su entorno y a las condiciones previamente modeladas por sus diseñadores y programadores. Los algoritmos son escritos por humanos y son estos quienes deciden las funciones de las máquinas y cómo estas son usadas.

A partir de ello, podemos señalar el contraste entre el razonamiento automático de la máquina y el razonamiento deliberativo característico del elemento humano. En palabras de Sharkey $(2019)^{21}$, dicho razonamiento propio del ser humano se conforma por un conjunto de procesos lentos que apuntan a un razonamiento reflexivo, de vital importancia para la toma de decisiones, y que requiere de recursos cognitivos como la memoria y la atención; mientras que el razonamiento automático que caracteriza a las máquinas presenta algunas dificultades cuando se aplica a sistemas de armas autónomas, ya que este tipo de razonamiento:

(1) Descuida la ambigüedad y suprime la duda: el razonamiento automático solo produce conclusiones, de manera que, si un objetivo se ve legítimo, será legítimo a pesar de las circunstancias. No hay búsqueda de interpretaciones alternativas o margen de incertidumbre, sino que una respuesta inequívoca aparece al instante.

(2) Infiere e inventa causas e intenciones: infiere rápidamente causas coherentes a partir de fragmentos de información disponible, es decir que ante una determinada circunstancia atribuye causas automáticamente, cayendo en un error sistemático a partir del razonamiento inductivo.

(3) Predisposición a la creencia y a la confirmación: favorece una aceptación de sugerencias no crítica y mantiene un fuerte sesgo.

(4) Se centra en la evidencia existente e ignora la ausencia de evidencia: construye explicaciones coherentes sin consideración de evidencia o información contextual.

En definitiva, la utilización de Laws representa grandes riesgos y peligros para la seguridad global y la estabilidad internacional. En los próximos párrafos, nombraremos y desarrollaremos los que a nuestro juicio merecen más atención.

Un efecto de la utilización de estos sistemas en el escenario internacional es la "proliferación". 22 Sin un límite al desarrollo, la prueba y producción de Laws, es probable que las mismas comiencen a proliferar de forma acelerada. Además, no todas las naciones tendrán la capacidad de llevar a cabo las revisiones pertinentes de modo que estos sistemas se adapten a los requerimientos del Derecho Internacional

\footnotetext{
${ }^{21}$ Miembro del International Committee for Robots Arms Control y de la Campaign to Stop Killer Robots.

${ }^{22}$ Los materiales necesarios para construir Laws se están volviendo cada vez más accesibles y baratos, aumentando de manera sustancial el riesgo de proliferación.
} 
Humanitario. Al respecto, un tema recurrente en la literatura sobre la adquisición de tecnologías avanzadas, y tal vez lejano, pero no por eso menor, es el problema que generaría que las Laws cayeran en manos de grupos terroristas. Si bien es necesario reconocer que para los grupos de estas características tener los recursos y conocimientos necesarios presenta grandes dificultades, las nuevas tecnologías siempre son una oportunidad, y no debe dejar de pensarse estos escenarios.

Otra de las consecuencias para tener en cuenta se refiere al "bajo costo para los conflictos armados”. Las Laws podrían conducir a acciones cortas de guerra, minimizando el uso de fuerzas militares humanas en las zonas de conflicto. Esto podría llevar a los Estados a utilizar la violencia sin realizar los procedimientos de consulta legales requeridos para desplegar tropas en un territorio. Los países se verían así seducidos a participar en más conflictos armados, a expensas de la desprotección de las poblaciones civiles. Por su parte -y como sucede actualmente- las operaciones encubiertas que requieran de la utilización de estos sistemas podrían aumentar exponencialmente.

La "interacción entre sistemas de armas" es una situación potencial que podría causar grandes accidentes en poblaciones civiles. Efectivamente, a medida que más países empleen Laws con "técnicas de enjambre" ${ }^{23}$, estas armas, como también el comando y los sistemas de control, se interrelacionarán inevitablemente. Y cuando algún dispositivo móvil controlado por software interactúe con dispositivos hostiles controlados por software desconocido, el resultado de dicha interacción sería científicamente imposible de predecir, y por ende es importante tener en cuenta su impacto en poblaciones civiles. El incremento de la mencionada interrelación entre sistemas autónomos y la velocidad de esa interacción impredecible, puede desencadenar un conflicto armado de manera accidental, antes de que el elemento humano tenga la posibilidad de reaccionar. La aceleración generada por el ritmo de batalla será tal, que la toma de decisiones humana no podrá ser lo suficientemente rápida. Debido a esto, las Laws requerirán el desarrollo de sistemas de respuesta defensivos más rápidos, y en este proceso se reducirá aún más el control humano sobre el campo de batalla.

Por otra parte, uno de los aspectos más importantes tal vez sea la posibilidad de que estos sistemas fallen o sean "vulnerados cibernéticamente". Al respecto, los humanos necesitan tener el control de los sistemas de armas para contrarrestar muchos de los potenciales peligros derivados del uso de armas totalmente informatizadas y autónomas. Riesgos tales como errores de codificación de software,

\footnotetext{
${ }^{23}$ Según Edwards (2000) el enjambre es una táctica de batalla en donde se realiza una maniobra que implica el ataque convergente de cinco (o más) unidades semiautónomas o autónomas en algún lugar determinado contra un objetivo específico (p .6-7).
} 
mal funcionamiento, degradación de las comunicaciones, ciberataques enemigos, infiltraciones en las cadenas de suministro industrial, intervención (jamming) o suplantación de identidad (spoofing), hacen de las Laws sistemas inseguros, fundamentalmente debido a los problemas que surgen de las comunicaciones y de la interoperabilidad que hacen a su seguridad digital.

Para finalizar, es preciso destacar que, si bien la superioridad que estos sistemas permiten detentar en un conflicto armado es relevante -como indica Headrick (2011), y aunque la tecnología proporciona a sus detentores poder sobre la naturaleza, ese poder -a la larga- puede volverse en contra de los mismos, y la tecnología per se no garantiza la victoria. El problema radica principalmente en que la posibilidad de proliferación y aumento en el uso de este tipo de armamento podría tener un gran impacto en las futuras formas de conflicto y, quizás, pudiera llevarnos hacia un escenario internacional más volátil. Al respecto, la idea de que la guerra podría convertirse en un deporte queda reflejada en la consigna de que las guerras sin riesgo pueden hacer prevalecer las soluciones militares por encima de las políticas.

\section{Conclusiones}

Podemos concluir que, con el desarrollo de las Laws, comienza a darse un paulatino proceso de "deshumanización de la guerra", a partir del cual la misma comienza a despojarse de sus características humanas. La robotización o automatización de los conflictos pueden llevarnos a escenarios donde el ocasional adversario ya no está al mismo nivel, es decir, donde la muerte ajena se convierte en algo totalmente banal y ya no existen las pautas comunes de interacción de una guerra convencional en términos de reconocimiento mutuo, sino que pasa a primar la indiferencia de la máquina, que carece de cualquier tipo de capacidad de compartir un entendimiento mutuo con quien sufre.

De ello se desprende el importante rol que el humano tiene en todo conflicto, fundamentalmente en cuanto a la selección de objetivos, como señala Scharre (2019), bajo tres puntos principales:1) el humano como operador esencial: el sistema de armas no puede ser preciso o tener compromisos efectivos sin el operador humano; 2) el humano como agente moral: el operador hace juicios basados en valores sobre si el uso de la fuerza es o no apropiado; 3 ) el operador humano como elemento de control ante eventuales fallas: el humano tiene la capacidad tanto de intervenir y alterar como de detener la operación del sistema de armas, en caso de que éste comience a fallar o de que cambien las circunstancias de manera abrupta (p. 13-15).

La idea de deshumanización no debe entenderse en términos peyorativos ni elogiosos. En este sentido, no debemos nublar el núcleo principal del argumento, referido al cambio revolucionario en la filosofía del combate que representa la 
introducción de estos sistemas en los conflictos armados modernos, en tanto, como muestra Spindel (2019), esto supone fundamentalmente: 1) un cambio en la distribución de los recursos humanos y tecnológicos necesarios para participar en la guerra y en las operaciones adyacentes a la misma; 2) un cambio en la velocidad de las operaciones, reduciendo el tiempo de toma de decisiones; 3) la disminución cada vez mayor de la presencia humana en el campo de batalla, permitiendo poner el foco en cuestiones más estratégicas en detrimento de las cuestiones de orden táctico (p. 6-7).

En cuanto a la discusión sobre los dilemas que implica la utilización de las Laws en el campo de batalla, rescataremos ocho parámetros propuestos por UNIDIR (2014, p. 5-7) para la realización de un abordaje que permita clasificar fielmente los peligros que estos sistemas representan:

(1) Funciones del arma: el control se debería basar en el conocimiento del sistema de armas, que contribuye asimismo a la evaluación de si el empleo de un arma es apropiado para un ataque particular. El aumento en la autonomía de las funciones críticas de las armas implica que la evaluación contextual sobre el objetivo y el ataque son delegadas en el propio sistema.

(2) Limitaciones espaciales: restringir el uso de determinados sistemas de armas en entornos específicos parece un mecanismo razonable para garantizar el control sobre tecnologías cada vez más autónomas, ya que de esta manera se garantiza un cierto grado de previsibilidad.

(3) Límites de tiempo: limitar el tiempo en que un sistema de armas puede operar es una manera adicional de ejercitar el control. Esto apunta a la idea de un umbral de tiempo en que un sistema puede operar con altos niveles de autonomía y de que, luego de ese tiempo establecido o del cumplimiento de la misión, el sistema regrese a un modo directamente controlado por el elemento humano.

(4) Previsibilidad: para que un sistema de armas esté bajo control, necesita comportarse de manera previsible en el entorno en el que se despliega y en la acción que está destinado a llevar a cabo. Una determinada acción puede ser previsible, pero ante la interacción con un entorno particular los resultados de esa acción pueden acabar teniendo consecuencias no deseadas.

(5) Distinción entre Sistemas contra Objetivos anti-materiales y Sistemas contra Combatientes, o que tengan consecuencias sobre la población civil: es posible que el grado de autonomía permitido para sistemas que apuntan a objetivos anti-materiales podría ser diferente a los diseñados para atacar personas; sin embargo, los sistemas de armas autónomos anti-materiales también pueden tener graves impactos sobre poblaciones civiles.

(6) Distinción entre Sistemas Estacionarios y Sistemas Móviles: se podría argumentar que es más aceptable dotar de autonomía a sistemas estacionarios (por 
ejemplo, aquellos que defienden una ubicación o instalación dada contra tipos específicos de amenaza), mientras que aquellos que son móviles cuenten con un mayor control humano para seleccionar objetivos; sin embargo, las primeras también deben tener el grado de distinción y capacidad para juzgar la proporcionalidad que se requiere según el Derecho Internacional Humanitario.

(7) Estándares de cumplimiento: dado que ningún sistema es 100\% predecible, cabe preguntarse si sería aceptable o legal desplegar un sistema de armas autónomo bajo el conocimiento de que sus acciones no serán del todo previsibles y qué consecuencias habría si alguno de ellos vulnerara el Derecho Internacional Humanitario.

(8) Verificación: una cultura de transparencia ofrecería oportunidades para el intercambio y el aprendizaje, y serviría como base para identificar principios comunes y de acercamiento entre los Estados. Estos esfuerzos pueden incluir iniciativas destinadas a abordar eficazmente las preocupaciones planteadas por las Laws a través de los compromisos convenidos entre los Estados en una declaración política, la elaboración de normas técnicas internacionales sobre pruebas, validación o verificación, así como moratorias nacionales sobre el desarrollo o la adquisición de Laws, e incluso medidas para apoyar la aplicación nacional de las normas convenidas internacionalmente.

Sin embargo, a pesar de lo expuesto, es posible sugerir, al igual que lo hace Freedman (2019), que las máquinas no nos caerán encima de forma tan repentina. Los avances en el campo de la tecnología deben superar aún muchas complicaciones. La aplicación de sistemas novedosos resulta mucho más lenta de lo que los entusiastas futuristas proclaman. Sabemos -porque el conocimiento del pasado nos lo permiteque las instituciones militares han solido oponerse a algunas innovaciones que ponían en peligro el total control humano sobre determinados instrumentos bélicos. ${ }^{24}$ Por su parte, la metamorfosis de las tecnologías más avanzadas en sistemas efectivamente operativos no ha resultado tan alentadora como parece: problemas de financiación, burocráticos o de ingeniería provocaron graves retrasos (p. 378-379). Por ende, los sistemas autónomos, tal vez, en el corto o mediano plazo serán complementarios más que reemplazantes de las plataformas no autónomas.

Lo que nos interesa recalcar (como punto final) es que la prohibición de estos tipos de sistemas es casi imposible. Como bien lo manifestamos en el trabajo, una vez que los Estados introducen estos armamentos y comienzan a ser utilizados prohibirlos se transforma en una tarea titánica, sin embargo, lo que se debe buscar es una regulación en su implementación. Un nivel como el actual, en donde los dispositivos

\footnotetext{
${ }^{24}$ En la década de 1950, el Mando Aéreo Estratégico mostró sus dudas respecto a que el emplazamiento de los misiles balísticos intercontinentales pudiera reemplazar a los bombarderos tripulados.
} 
más autónomos y menos supervisados no son la regla representa un buen punto de partida.

\section{Referencias bibliográficas}

Adorno, T. (2001). Minima Moralia, Taurus.

Arendt, H. (2006). Sobre la Violencia, Alianza.

Beck, U. (2009). La Sociedad del Riesgo Mundial: en busca de la seguridad perdida, Colección Estado y Sociedad, Paidós.

Bostrom, N. y Circovic, M. (2008). Global Catastrophic Risks, Oxford University Press.

Chan, M. (2019). China and the U.S. are fighting a major battle over killer robots and the future of AI. Time. Recuperado de https://time.com/5673240/china-killerrobots-weapons/

Coker, C. (2013). Warrior Geeks: how $21^{\text {st }}$ century technology is changing the way we fight and think about war, Oxford University Press.

Colom, G. (2015). Rumsfeld Revisited: La Tercera Estrategia de Compensación $\begin{array}{llll}\text { estadounidense, } & \text { Revista } & \text { UNISCI } & \text { 69-88. }\end{array}$ https://dx.doi.org/10.5209/rev_RUNI.2015.n38.496435

Department of Defense (2012). Autonomy in Weapon Systems. (3000.09). Recuperado de https://www.esd.whs.mil/ portals/54/documents/dd/issuances/dodd/300009p.pdf

Docherty, B. y Griechen, M. (2019). Legal Arguments against Autonomous Weapons en Hunt, Erin (2019): Campaign to Stop Killer Robots (campaigner's kit), Campaign to Stop Killer Robots, p.p. 7-9.

Edwards, S. J. A (2000). Swarming on the Battlefield: Past, present and future. En Rand Corporation.

Freedman, L. (2019). La guerra futura. Un estudio sobre el pasado y el presente, Ed. Crítica. Fritsch, S. (2017). Technology, Conflict and International Relations. En Yetiv, S. y James, P. (Ed), Advancing Interdisciplinary Approaches to International Relations, p.p. 115-151. Palgrave McMillan. DOI 10.1007/978-3-319-40823-1

Gusterson, H., (2014). Towards an Anthropology of Drones: Remaking Space, Time, and Valor in Combat, en Evangelista, M. y Shue, H. (Ed.) The American Way of Bombing. Changing Ethical and Legal Norms, from B-17s to Drones, Nueva York: Cornell University Press, p.p. 191-206.

Gusterson, H. (2016). Drone: Remote Control Warfare. Cambridge, Massachusetts; London, England: The MIT Press.

Headrick, D. R. (2011). El Poder y el Imperio: la Tecnología y el Imperialismo de 1400 a la actualidad, Serie Mayor, Ed. Crítica.

Human Rights Watch \& IHCR. (2013). Review of the 2012 U.S. policy on autonomy in weapon systems. Human RightsWatch and Harvard Law School International 
Human Rights Clinic. Recuperado de https://www.hrw.org/news/2013/04/15/review-2012-us-policy-autonomyweapons-systems

International Committee of The Red Cross (2014). Autonomous Weapon Systems: technical, military, legal and humanitarian aspects. Recuperado de https://www.icrc.org/en/document/report-icrc-meeting-autonomousweapon-systems-26-28-march-2014

International Committee of The Red Cross (2005). Derecho Internacional Humanitario: respuestas a sus preguntas. Recuperado de CICR-Respuestas-ESP.qxd (icrc.org)

International Committee of The Red Cross (2021). ICRC position on autonomous weapon systems. Recuperado de

Joint Doctrine Note 0-30.2 (2017). Unmanned Aircraft Systems. Recuperado de https://assets.publishing.service.gov.uk/government/uploads/system/uploads /attachment_data/file/673940/doctrine_uk_uas_jdp_0_30_2.pdf

Joint Doctrine Note 2/11(2011). The UK Approach to unmanned Aircraft Systems. Recuperado de https://www.law.upenn.edu/live/files/3890-uk-ministry-ofdefense-joint-doctrine-note-211-the

Kayzer, D. y Denk, S. (2017). Keeping Control. European positions on lethal autonomous weapon systems, PAX. Recuperado de https://www.paxforpeace.nl/media/files/pax-report-keeping-control.pdf Keegan, J. (2013). El Rostro de la Batalla, Turner Noema.

Kozyulin, V. (2019). A Russian Perspective. Militarization of AI (PRI Center - Russian Center for Policy Research), en The Militarization of Artificial Intelligence (2019), Stanley Center for Peace and Security | UNODA | Stimson.

Martinez, V. y Rodríguez R. (2020). Aportes al debate del uso de la Inteligencia Artificial para aplicaciones armamentistas: una mirada desde el sur. En SEHLAC.

Pax (2019). The state of IA. Recuperado de https://www.paxforpeace.nl/publications/all-publications/the-state-of-ai

Ryan, K. (2014). What is wrong with Drones? The Battlefield in International Humanitarian Law, en Evangelista, M. y Shue, H. (ed.) The American Way of Bombing. Changing Ethical and Legal Norms, from B-17s to Drones, Nueva York: Cornell University Press, pp. 207-223.

Yousef Sandoval, L. (2018). Aviones y drones: el elemento aéreo, en Carl Schmitt. Daimon Revista Internacional De Filosofia, (N73), 167-180. https://doi.org/10.6018/daimon/258711

Scharre, P. (2019). Military Applications of Artificial Intelligence: potential risks to International Peace and Security, en The Militarization of Artificial Intelligence (2019), Stanley Center forPeace and Security | UNODA | Stimson. 
Schmitt, C. (2005). El nomos de la tierra en el derecho de gentes del Ius Publicum Europaeum, Struhart.

Sharkey, N. (2019). Human Control of Weapons Systems, en Hunt, E. (2019). Campaign to Stop Killer Robots (campaigner's kit), Campaign to Stop Killer Robots.

Sloan, E. (2015). Robotics at War, Survival, Vol.57 (2), International Institute for Strategic Studies, p.p. 107-120. https://doi.org/10.1080/00396338.2015.1090133

Spindel, J. (2019). Artificial Intelligence, nuclear weapons, and Strategic Stability, en The Militarization of Artificial Intelligence (2019), Stanley Center for Peace and Security | UNODA | Stimson.

United Nations Institute for Disarmament Research (UNIDIR). (2014). The Weaponization of Increasingly Autonomous Technologies: considering how meaningful human control might move the discussion forward, UNIDIR resources $\mathrm{N}^{\circ} 2$. Recuperado de https://unidir.org/files/publications/pdfs/consideringhow-meaningful-human-control-might-move-the-discussion-forward-en615.pdf 